\title{
Exploring the Possibilities of Body Motion Data for Human Computer Interaction Research
}

\author{
Johann Schrammel ${ }^{1}$, Lucas Paletta ${ }^{2}$, and Manfred Tscheligi ${ }^{1,3}$ \\ ${ }^{1}$ CURE - Center for Usability Research \& Engineering \\ Modecenterstrasse 17, 1110 Wien, Austria \\ \{schrammel, tscheligi\} @cure.at \\ ${ }^{2}$ Joanneum Research \\ Wastiangasse 6, $8010 \mathrm{Graz}$, Austria \\ lucas.paletta@joanneum.at \\ ${ }^{3}$ HCI-Unit, ICT\&S, Universität Salzburg \\ Sigmund-Haffner-Gasse 18, 5020 Salzburg, Austria
}

\begin{abstract}
The ability to move is an important characteristic of the human condition and an important aspect for interactive settings. The role of body movement however was not addressed with priority in human computer interaction until now. In this paper we explore the possibilities and issues for usability and user experience research utilizing body motion data. We provide an overview of relevant related work and report the setup and initial results of two studies utilizing body motion capture. We discuss the experiences made in using motion capture approaches for human computer interaction research and provide an outlook on future directions of research.
\end{abstract}

\section{Introduction}

The possibility to move is an important aspect of the human condition, and body motion is a natural and essential element of interactive settings and scenarios. Until now the role of body movement however was not addressed with priority in human computer interaction and user experience research. This relative lack of interest probably can be explained by the limitations of available technologies capable of collecting motion data reliably and economically. Recently these technological restrictions have become less and less severe, and in future we expect an almost complete removal of restrictions stemming from technical limitations. More and more tools become available that allow to easily access and utilize body movements for interaction purposes. Until recently game controllers for example only used the tiny movements of the fingers to control the action, and current office applications rely almost exclusively on mouse and keyboard input. This is about to change, as the example of the huge success of the Nintendo Wii indicates.

The main goal of this paper is to explore the resulting possibilities and issues for usability and user experience research. We firstly want to outline the theoretical background and present related research focusing on existing work on capturing motion data, the role of movement in human computer interaction. Secondly we present two 
examples using different technological approaches in addressing these issues and provide information on how we used these approaches in human computer interaction research. We discuss the possibilities, advantages, shortcomings and research implications of these approaches. Finally we provide thoughts on future direction of research using body motion detection and open issues to be solved.

\section{Capturing Motion Data}

Eadweard Muybridge introduced motion capture 1887 in his now famous experiments entitled Animal Locomotion, in which he studied the way in which animals and birds moved by taking series of photographs. Driven by similar interests in 1973 psychologist Johansson conducted his Moving Light Display (MLD) experiments. Johansson attached reflective markers to the joints of test subjects and recorded their motions [1]. He then asked subjects to identify known movements after being shown only the trajectories of the markers. Since then numerous different approaches to capture and analyze human motion have been suggested, developed and commercially used. This high interest is driven by numerous promising application domains for motion capture e.g. advanced user interfaces, motion analysis for clinical and sports training purposes, surveillance systems or virtual reality applications. Motion capture systems have been developed using very different types of technologies.

Vision based techniques capture body movement by use of cameras, either using markers or working markerless. Vision-based human motion estimation and analysis has been a thriving area of research within the past years. In an overview article Moeslund [2] for example identified over three-hundred related publications over the period of 2000-06 in major conferences and journals dealing with this topic. In a recent special issue of the Int. Journal of Computer Vision on the topic of human motion tracking Sigal and Black [3] characterized it as a "mostly solved problem in constrained situations". However, in unconstrained contexts and for real-time applications there are still important issues to be adressed.

Another approach uses feedback from angle sensors attached to the human body joints. Such systems are commercially available, provide reliable data and have been used successfully in HCI-studies [e.g. 4, 5]. However, they are rather cumbersome and obtrusive for the user. The latest model of the GYPSY-6 exoskeleton by Animazoo for example still weights over $6 \mathrm{~kg}$ and takes about 10 minutes to setup.

A third approach uses data from position, orientation and accelerometer sensors attached to the human body. Knight et al. [6] report various uses of accelerometers ranging from physics teaching, science education, posture measurement and ambulatory monitoring. Examplary applications within the field of HCI are Shoogle [7], DJogger [8] or ubifit [9]. The advantage of this tracking approach is that the used sensors are rather cheap, easy to track and relatively unobtrusive for the user. However, the information these sensors provide on body motion is typically either not very accurate (position) or provides only relative data (accelerometers), and therefore is more difficult to use and interpret than complete motion information. A popular implementation variant due to its ease of implementation for this approach is to utilize the controllers of the Nintendo Wii as accelerometer sensors [e.g. 10, 11, 12, 13 ]. 
Next we also want to briefly mention approaches that focus only on capturing single elements of body motions. For example big size touch screens such as used in tabletop computing could be considered as type of motion capture devices as they have the capability of tracking the position of the body part in contact with the screen (typically the fingers) [e.g. 14, 15, 7].

Summarizing we can say that cheap controllers and sensors that allow to recognize and track the movement of users have become available, and that they are more and more used in dedicated interaction devices. Examples include accelerometer based (e.g. Nintendos Wii) or computer vision based (Microsofts' project Natal) game controllers. Also, more and more mobile devices such as handheld phones or tablet computers are equipped with build-in accelerometer and tilt sensors, and therefore allow to be used as motion controllers and enable new interactive experiences. Another driver fostering increased importance of movement in interaction is the continuing success of touch-based interaction combined with decreasing costs of big-size screens. This two effects lead to the inclusion of semi-natural and intuitive body and movement-gestures in the interaction. Furthermore progress in gesture recognition and mobile augmented reality applications fosters the application possibilities of whole body interaction.

We think that these developments drive interaction towards more and more involvement of movement resulting in interaction styles that could be summarized as whole body interaction.

\section{Analyzing Motion Data}

In many cases the available motion data can be directly used for interaction purposes e.g. if the movement and position of the users lower arm is tracked correctly it can be used as input device e.g. as a pointer. However, human motion data contains more information than the position and orientation of body parts alone. Analyzing movement patterns across time researchers have identified several possibilities to interpret the data in a semantically meaningful way.

A first popular direction of analysis is the automated detection and recognition of user behavior and activity. Research has shown that state-of-the-art systems are able to successfully detect different types of user behavior and activities such as walking, running, standing, and sitting based on their specific movement patterns [e.g. 16, 17, 9]. Also everyday activities such as brushing ones teethes or vacuuming the floor could be learned and detected successfully by these systems [18].

However, it is not only possible to detect physical activities of the user or his context, motion data also allows to derive data about the cognitive and emotional state of users. [19] showed that humans are capable of judging the emotional state of a human body from motion information alone. Similarly [20] showed that it is possible to recognize emotions better than chance based solely on gait information. Since then efforts have been made to automatically detect and estimate the emotional state of users based on motion data (more details on the relationship between motion and emotion are discussed in the next chapter). It has also been shown that personality traits correlate with characteristics of music-induced movement [21], suggesting that motion data can bee used to estimate personality traits. 
Another possible application that has been addressed in research is the usage of the uniqueness of movement patterns of a user for authentication purposes. Applications in the context of security and disambiguation between users in multi-user contexts have been proposed. For example gait recognition has been successfully demonstrated [e.g. 22].

\section{Motion, Emotion, Interaction and Experience}

The previous paragraphs showed that from a viewpoint of analysis and application scenarios several relevant directions exist. To fully utilize the potential of these analyses it is helpful to also take a phenomenological view on the different functions of body movements. For the context of interactive scenarios in general and gaming in specific Berthouze [23] distinguishes between five different types or functions of body movements:

Table 1. Categorization of body movement types as proposed by Berthouze [23]

\begin{tabular}{|l|l|}
\hline Body movement & Description \\
\hline Task-control & necessary to control the game \\
\hline Task-facilitating & facilitating the control \\
\hline Task(role)-related & $\begin{array}{l}\text { typical of the role defined by the game } \\
\text { scenario }\end{array}$ \\
\hline Enjoyment and emotional expressions & $\begin{array}{l}\text { expressing affective states related to or } \\
\text { induced by the game experience }\end{array}$ \\
\hline $\begin{array}{l}\text { Social behavior (attention seeking, } \\
\text { synchronized movement, etc.) }\end{array}$ & supporting social interaction \\
\hline
\end{tabular}

Taken a closer look, Table 1 above shows that important aspects of the functionality of body motions are related to the expression of emotional experiences. Motion and Emotion are even very similar literally, and researchers suspect that there are causal relations between these two concepts. The direction of this causality and the details of the involved mechanisms are not clear yet and subject to ongoing research.

A study by Riskind and Gotay [24], for example, revealed how "subjects who had been temporarily placed in a slumped, depressed physical posture later appeared to develop helplessness more readily, as assessed by their lack of persistence in a standard learned helplessness task, than did subjects who had been placed in an expansive, upright posture."

With regard to the relationship between movement and user experience common sense and results from UX research suggests that the interaction with products, tools, and artifacts can be enriched by allowing people to move naturally and unrestrictedly $[4,25]$. If people can express themselves using their whole body, they immerse into another world more naturally and easily. Results show that even engagement and social interaction are encouraged by controllers that afford movement [26]. Izard et al. [27] describes neural, sensorimotor, motivational and cognitive processes that can influence emotions. Body posture and movement can activate emotions by afferent processes. 
These examples of the important role of motion for experience in interactive settings highlight the need to include data on this aspect in user experience research and to further research the involved processes in more detail.

\section{Example Studies Utilizing Body Motions}

In the previous chapters we analyzed that utilization of motion data and embodied interaction from a technological and theoretical viewpoint. We conclude that there seems to be a high potential to enhance interactive experiences by including motion data in interaction design and user experience. In the next sections we want to present two different approaches we have used in praxis for studying user experience and human computer interaction aspects considering motion. We briefly report selected results of this studies to show the possibilities of the chosen approaches. However, the full analysis of data and the discussion of the individual results are not within the scope of this paper and are subject of (future) dedicated publications.

\section{Study 1: Using accelerometer data to track users' movement during gameplay}

A first setting we want to describe to be able to discuss and analyze the possibilities of motion capture for user experience research is in the context of game research.

Research Questions. In detail we were interested in answering the question whether a more embodied way of controlling the interaction is amplifying experience in a gaming context as expected?

Study Design. To control weather our hypothesis of enhanced experiences is correct we conducted a simple comparison of self-reported experiences in two different gaming conditions either controlling a game by Wii-controllers designed to be used with body gestures and traditional joystick-like controllers using only the thumbs to control the game.

Six female and six male subjects participated in the study. Participants were between 20 and 36 years old and had extensive experience in computer game playing. Each participant brought a friend of the same sex along, against whom they were playing in the gaming session of the study.

Participants were playing two different tasks (food tracing and cow tossing) from the game "Rayman Raving Rabbids" on the Sony Playstation 2 (seated condition) and on the Nintendo Wii (motion condition). Play time to finish the tasks on either type of console typically was between 10 and 15 minutes. Amount of movements of the player was tracked by use of a second Wii Remote attached to the dominant arm (see below for detailed description of setup). Participants played against the friend they brought with them. "Rayman Raving Rabbids" has very similar graphics on the two consoles and gaming sites give them the same rating. Half of the participants started with the Wii, and half with the Ps2.

After each condition participants were asked to rate their average experience during playing by use of Emocards [28]. Additionally, participants were asked to describe the emotionally most outstanding situation during play. They were also asked to fill out a questionnaire using Likert-scale items regarding perceived agitation and emotions. 
Additionally facial muscle activity of the zygomaticus major muscle (the "smile" muscle) and the currogator supercilii muscle (the "frown" muscle) was measured by use of electromyography to gather continous uindicators for user experience.

Motion capture setup: For Capturing the motion of the users we used Wiimotes from Nintendo. Wiimotes are equipped with three-axis accelerometer sensors, and they are ideal for rapid deployment since they are equipped with wireless connection functionality to a host computer using Bluetooth, and free open source code for accessing the sensor data exists. In detail we used the DarwiinRemote (Version 0.3.1) Software (available at http://sourceforge.net/projects/darwiin-remote/). As the Wiimotes communicate using Bluetooth it is unfortunately not possible to read out the movement data of a Wiimote and to use it at the same time as a controller in the standard Wii configuration. The Wiimotes are also equipped with an infrared camera, a vibration sensor, and a loudspeaker. These are not used for the experiments reported here, but present interesting possibilities for usage in future projects. Figure 1 below shows the readings of the accelerometer-sensors provided by DarwiinRemoteSoftware. In the pictured sequence the Wiimote was first moved back and forth along all three main dimensions resulting in the dominance of the data from the regarding accelerometer, and then wiggled without specific direction. To use the data in analysis the readings from all sensors can be recorded in a time-stamped logfile.

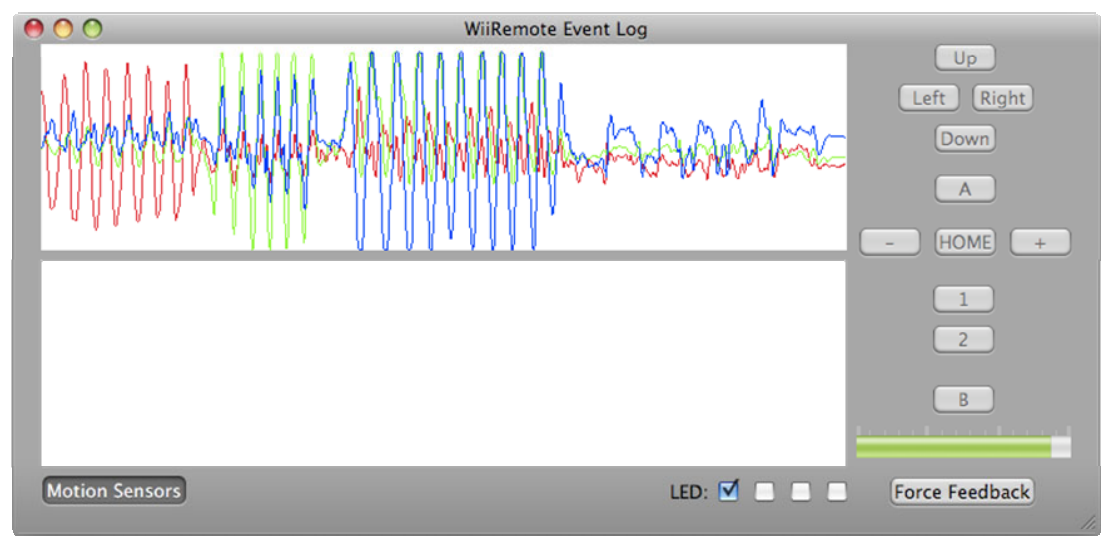

Fig. 1. Screenshot from DarwiinRemote-Software Showing Readings from Accelerometers

Comparing Gaming Conditions: Users reported more positive experience when using the Wii compared to the Ps2. Two-way repeated measures ANOVA with sex and game condition as independent variables showed a significant main effect for self-reported experience.

Correlation of Motion and EMG: Situational motion data (as provided by the Wii) and experience indicators (EMG data) was correlated to further analyze the relationship of these two data sources. The motions of gamers show a highly significant correlation with both, the EMG-values of the zygomaticus $(r=0.139, p<0.01)$ and the 
currogator $(\mathrm{r}=0.256, \mathrm{p}<0.01)$. One might suspect that this correlation is caused by a slight activation of the tracked muscles related to an overall increased tension during movements. However, several arguments point against this interpretation. First, in this analysis only a binary representation of EMG data was used and threshold values were manually assigned to the data taking care they are set high enough to only detect meaningful activation. Second, several random samples of video data from different study participants were analyzed regarding the plausibility of the binary EMGoutputs. No indication for erroneous data was found.

Qualitative results support this interpretation, as the following example statements from participants show:

"Playing on the Wii is more fun, the whole body is in movement and not only fingers and thumbs."

"I preferred playing on the Wii better. You have to do more with the arm and move more. If you are allowed to do this, you can better feel the game play"

\section{Study 2: Using position data to study the relationship of users' attention and motion in public outdoor scenarios}

Research Questions. The main question of the second study was to better understand, which areas and objects receive attention by pedestrians in a shopping street, and whether there is a significant influence of the users movement on the direction of attention.

Study Design. 16 study participants where equipped with mobile eye-tracking systems and various sensors (detailed description below) which were used to track the users motion. Participants were asked to perform a realistic task within the inner city of Graz, which was to stroll through the Grazer Herrengasse and inform themselves about shopping possibilies in the street. Overall time of data capture per test person was about 15 minutes.

Motion capture setup. In this study a different motion capture setup was chosen. For capturing the users body and head movements two low-cost Inertial Measurement Units (IMUs) of manufacturer XSens (http://www.Xsens.com) were used. Xsens MTi$\mathrm{G}$ was used to capture head data (the sensor was mounted to the helmet of the eyetracking equipment), and Xsens MTx was used to capture body movement (the sensor was attached to the participants' waist using a customary belt pouch). Both sensors were connected via USB to a Laptop-Computer the test participant carried in a rucksack. "MT Manager"-Software provided by the manufacturer of the sensors was used to record the data.

We also used the build-in sensors of the Google Nexus One smartphone to capture GPS-position data. The position fixing component of the device is a GPS receiver, which is integrated directly within the chipset. Unfortunately, the manufacturer does not provide publicly available specifications. The device is also equipped with integrated accelerometers and a tilt compensated magnetic sensor. Only the GPS-data was used as the X-sensors provide more accurate data. However, using the build-in sensors would be a very attractive option for application scenarios that require only tracking the body position and do not call for very high accuracy. 
Additionally a mobile eye-tracking system was used during the study. This analysis of this data is still underway, and results with regard to gaze fixations are not yet available and will be the subject of future publications. Figure 2 below shows a photograph of a participant equipped with the full set of sensors.

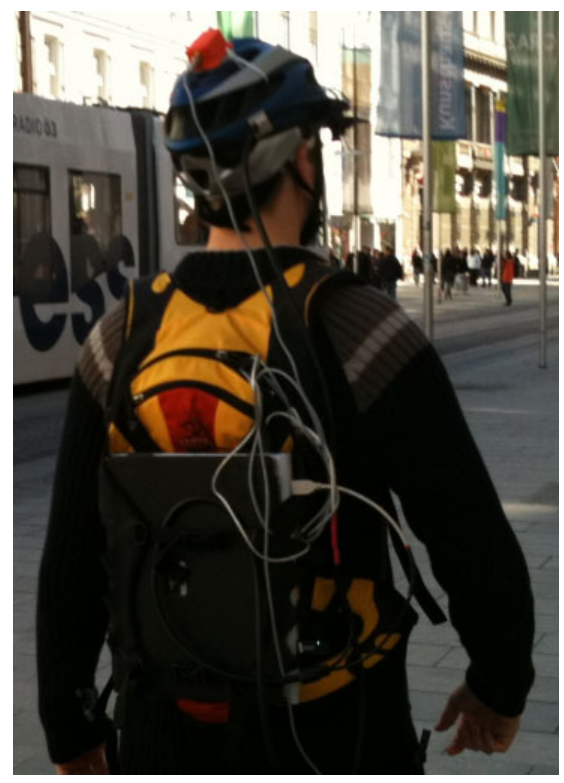

Fig. 2. Participant equipped with tracking technology in the Grazer Herrengasse

Body and Head Orientation of Pedestrians: A first focus of analysis of the data was to analyze the area of the attention of the pedestrians in the urban setting. Our basic reference system for the field of attention is the human body itself. Regarding horizontal direction of attention the reference is the body orientation of the user (measured by the sensor at the waist), and the relative orientation of the head (measured by the sensor mounted on the helmet). For vertical orientation we chose to use the perpendicular axis as main reference. Figure 3 provides an overview of the average horizontal orientation of the users head relative to the body orientation. The graphic shows a distribution of orientation as might be expected: In the majority of cases the head orientation is aligned with the body orientation, with decreasing numbers for relative orientations to the sides. There are almost no cases with a relative angle of more than 60 degrees in either direction.

Figure 4 shows the results for the vertical alignment of the head of pedestrian users on the move. The image clearly shows that the most attention is directed towards a slightly lowered position, and that there is much more attention towards the lower sphere than the upper sphere. This can easily be explained by the needs of pedestrians to navigate between other pedestrians, the usage and consultation of personal devices (e.g. looking on the watch, consulting written materials, using electronic devices, etc.) and watching objects of interest in shopping windows. 


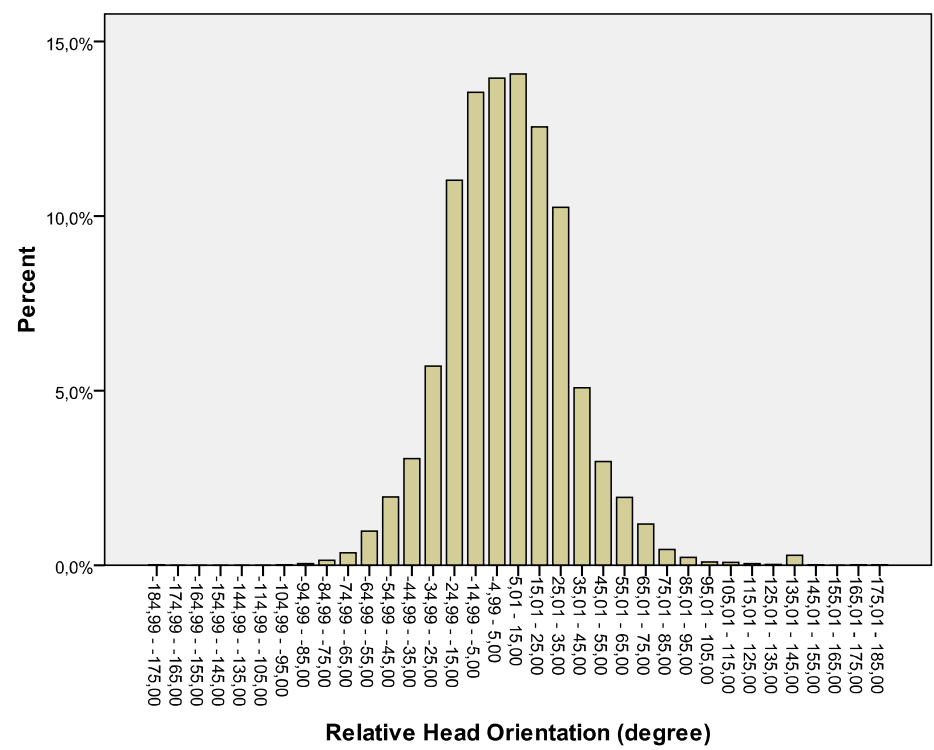

Fig. 3. Relative horizontal head orientation expressed in degrees across all test participants

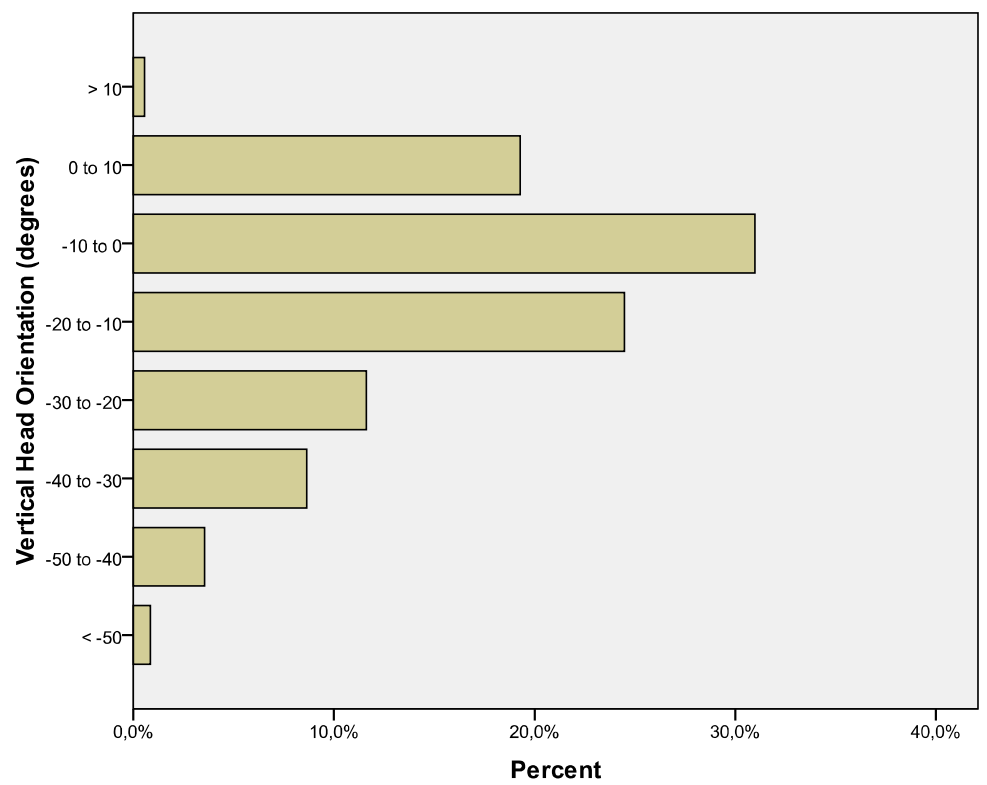

Fig. 4. Relative vertical head orientation expressed in degrees across all test participants 
Influence of Movement Velocity on Body and Head Orientation: We next were interested in analyzing whether there is an influence of the users movement velocity on the field of attention as expressed by relative head orientation. Our hypothesis was that the relative horizontal head orientation is directed stronger towards the front as the user has to increasingly take care of possible obstacles the faster he moves. As a first initial indicator for such a relationship we calculate regression (Pearson) between the movement velocity and the absolute value of the relative horizontal head orientation. We found a highly significant correlation with $r$ being -0.154 . The faster the user moves the less he is looking to his sides.

\section{Discussion}

In the above sections we described the application of motion tracking technology in two different studies. In this section we want to discuss the individual results of the studies in much detail but focus on the experiences made with the different approaches and discuss the possibilities of tracking technologies for HCI research.

Both study setups show that the utilization of motion capture provides valuable data for the research. The finding of study 1 directly support our hypothesis that increased involvement of motion provides positive chances for enhanced user experiences, and study 2 showed that tracking that can provide helpful information of the users attention, that would not be available otherwise.

Practical issues. A first experience from applying motion tracking technology for human computer interaction research is that setup is rather easy and can be done by non-specialists people with basic computer skills without problems. Also, cost of tracking devices is not an obstacle, as the required sensors are rather cheap. Setuptime and data extraction definitely takes some extra time and precautions, however compared to the gain in data it is a rather small effort that is required. In detail initial effort to setup the tracking systems was less than a day (including testing of the equipment) for both setups. The effort during running studies is also very small, as the needed effort is mainly in attaching the additional sensors and starting data recording.

A practical aspect that needs special considerations is the synchronization of data. Currently available capturing software does not yet support the integration with other tools used in research very well, and synchronization of data has to be done based on time-stamped raw data. For ease of synchronization we therefore recommend to use the same sampling frequency in all data recording channels, and to follow god practice of labeling data files and variables.

The interpretation of data is a main issue when using motion data. Automated behavior and context detection and interpretation is a very active field of research. However, there are no off-the-shelf solutions available yet that are easy to use by nonexperts and provide reliable data. Typically, the available approaches and algorithms need to be specifically targeted to the used context of the study, and extensive calibration and fine-tuning of parameters by experts is needed to achieve good results. However, there are several aspects of body motion that can be easily interpreted, do not need extensive setup and anyhow provide valuable data. In Study 1 we showed that accelerometer data and the conveyed information about the amount of movement can 
be helpful for a better understanding of gaming experience, and study 2 showed that basic movement data such as velocity and orientation allow to better understand patterns of attention of pedestrians in urban outdoor settings.

On very big advantage of using motion data is the availability of continuous and unobtrusively captured user data. This allows to study user behavior and experience related to the sensed variables across time, without the need to interrupt the user with frequent questions and thereby artificially altering the interactive process. Also such setups allow the study facilitator to observe data online, and to take targeted interventions if required by the study goals or to asked focused questions in follow-up interviews referring to the specifics of a situation brought to attention of the researcher by the online data. This aspect will be especially interesting once reliable detection mechanisms become available, however already now with very unreliable data or only human interpretation this capturing mechanisms could form a good basis for analysis. Another interesting possibility for research is to use the recorded data to identify sequences of special interest and to discuss these situations with participants after the test.

A further advantage of utilizing body motion data in HCI-research is the possibility to easily compare the behavior of different study participants with regard to their interaction styles. The availability of recorded data fosters the comparative analysis either by use of statistical procedures or by the visual analysis of movement patterns.

Another relevant application context for body motion data is the facilitation of Wizard-of-Oz studies. In the section on related work numerous possibilities for the application of body motion data in different types of interfaces has been shown, and research is needed on how to optimally design these interfaces. Wizard-of-Oz studies with a human operator simulating the targeted behavior of different algorithms could help to identify the most valuable directions of research early in the research process. Visually displaying the readings of motion sensors could very much ease the task of the wizard, which is known to be a very challenging responsibility.

To even more broaden the possibilities of motion data in future research we want to especially focus on the following areas:

- Include eye tracking data and integrate with motion data to generate unified method for studying users' attention in outdoor scenarios. We also work on integrating this model with a virtual model of the environment.

- Expand classification of body movement types to more general scenarios and framework for understanding the meaning and function of body movements.

- Develop set of methods that allows recognizing relevant behavior for HCI automatically. We do not expect to achieve $100 \%$ accuracy, but preprocessing of data will enormously speed up the annotation of observation data.

\section{Conclusions}

In this paper we showed the relevance and potential of body motion data for human computer interaction research and provided examples from real studies in the field of HCI. The approaches we described and discussed can and easily be used by other researchers, and valuable inputs for $\mathrm{HCI}$ can be generated. 


\section{References}

1. Johansson, G.: Visual Perception of Biological Motion and a Model for Its Analysis. Perception Psychophysics 14(2), 201-211 (1973)

2. Moeslund, T.B., Hilton, A., Krüger, V.: A survey of advances in vision-based human motion capture and analysis Comput. Image Underst. 104(2), 90-126 (2006)

3. Sigal, L., Black, M.J.: Guest Editorial: State of the Art in Image- and Video-Based Human Pose and Motion Estimation. Int. J. Comput. Vision 87(1-2), 1-3 (2010)

4. Bianchi-Berthouze, N., Kim, W.W., Patel, D.: Does Body Movement Engage You More in Digital Game Play? and Why? In: Paiva, A.C.R., Prada, R., Picard, R.W. (eds.) ACII 2007. LNCS, vol. 4738, pp. 102-113. Springer, Heidelberg (2007)

5. Pasch, M., Berthouze, N., van Dijk, E., Nijholt, A.: Motivations, Strategies, and Movement Patterns of Video Gamers Playing Nintendo Wii Boxing. In: ECAG 2008 (2008)

6. Knight, J.F., Bristow, H.W., Anastopoulou, S., Baber, C., Schwirtz, A., Arvanitis, T.N.: Uses of accelerometer data collected from a wearable system Personal Ubiquitous Comput., vol. 11(2), pp. 117-132. Springer, Heidelberg (2007)

7. Williamson, J., Murray-Smith, R., Hughes, S.: Shoogle: excitatory multimodal interaction on mobile devices. In: Proceedings of the SIGCHI conference on Human factors in computing systems, CHI 2007, pp. 121-124. ACM, New York (2007)

8. Biehl, J.T., Adamczyk, P.D., Bailey, B.P.: DJogger: a mobile dynamic music device. In: CHI 2006 extended abstracts on Human factors in computing systems, CHI 2006. ACM, New York (2006)

9. Consolvo, S., McDonald, D.W., Toscos, T., Chen, M.Y., Froehlich, J., Harrison, B., Klasnja, P., LaMarca, A., LeGrand, L., Libby, R., Smith, I., Landay, J.A.: Activity sensing in the wild: a field trial of ubifit garden. In: Proceeding of the twenty-sixth annual SIGCHI conference on Human factors in computing systems, CHI 2008, pp. 1797-1806. ACM, New York (2008)

10. Hafner, V.V., Bachmann, F.: Human-Humanoid Walking Gait Recognition Humanoids 2008. In: 8th IEEE-RAS International Conference on Humanoid Robots (2008)

11. Schou, T., Gardner, H.J.: A Wii remote, a game engine, five sensor bars and a virtual reality theatre. In: Proceedings of the 19th Australasian conference on Computer-Human Interaction, OZCHI 2007, pp. 231-234. ACM, New York (2007)

12. Schlömer, T., Poppinga, B., Henze, N., Boll, S.: Gesture recognition with a Wii controller. In: Proceedings of the 2nd international conference on Tangible and embedded interaction, TEI 2008, pp. 11-14. ACM, New York (2008)

13. Schreiber, M., Wilamowitz-Moellendorff, M., Bruder, R.: New Interaction Concepts by Using the Wii Remote. In: Proceedings of the 13th International Conference on HumanComputer Interaction. Part II, pp. 261-270. Springer, Heidelberg (2009)

14. Patten, J., Recht, B., Ishii, H.: Interaction techniques for musical performance with tabletop tangible interfaces. In: Proceedings of the 2006 ACM SIGCHI International Conference on Advances in Computer Entertainment Technology, ACE 2006, p. 27. ACM, New York (2006)

15. Collins, A.E.: Exploring tabletop file system interaction. In: CHI 2007 Extended Abstracts on Human Factors in Computing Systems, CHI 2007, pp. 2171-2176. ACM, New York (2007)

16. Ravi, N., Dandekar, N., Mysore, P., Littman, M.L.: Activity recognition from accceleration data American Association for Artificial Intelligence (2005)

17. Györbiro, N., Fabian, A., Homanyi, G.: An activity recognition system for mobile phones Mob. Netw. Appl. 14(1), 82-91 (2009) 
18. Yang, J.-Y., Wang, J.-S., Chen, Y.-P.: Using acceleration measurements for activity recognition: An effective learning algorithm for constructing neural classifiers. Pattern Recognition Letters 29(16), 2213-2220 (2008)

19. Dittrich, W.H., Troscianko, T., Lea, S.E., Morgan, D.: Perception of emotion from dynamic point-light displays represented in dance. Perception 25 (1996)

20. Montepare, J.M., Goldstein, S.B., Clausen, A.: The identification of emotions from gait information. Journal of Nonverbal Behavior 11 (1987)

21. Geoff, L., Saarikallio, S., Toiviainen, P.: Personality traits correlate with characteristics of music-induced movement. In: 7th Triennial Conference of European Society for the Cognitive Sciences of Music, ESCOM 2009 (2009)

22. Gafurov, D., Snekkenes, E.: Gait recognition using wearable motion recording sensors. EURASIP J. Adv. Signal Process, Hindawi Publishing Corp. 2009, 1-16 (2009)

23. Berthouze, N.: Body movement as a means to modulate engagement in computer games. In: Workshop on Whole body Interaction II: The future of the human body, HCI 2008 (2008)

24. Riskind, J.H., Gotay, C.C.: Physical posture: Could it have regulatory or feedback effects on motivation and emotion? Motivation and Emotion 6 (1982)

25. Moen, J.: From hand-held to body-worn: embodied experiences of the design and use of a wearable movement-based interaction concept. In: Proceedings of the 1st International Conference on Tangible and Embedded Interaction, TEI 2007, pp. 251-258. ACM, New York (2007)

26. Lindley, S.E., Le Couteur, J., Berthouze, N.L.: Stirring up experience through movement in game play: effects on engagement and social behavior. In: Proceeding of the CHI 2008 SIGCHI Conference on Human Factors in Computing Systems. ACM, New York (2008)

27. Izard, C.: Four Systems for Emotion Activation: Cognitive and Noncognitive Processes. Psychological Review 100 (1993)

28. Desmet, P., Overbeeke, C., Tax, S.: Designing products with added emotional value: development and application of an approach for research through design. The Design Journal 4(1) (2001) 